Laura Rubio - Teresa Moreno - Juan Pablo

Lamas · Carmen García-Jares · Marta Lores

\title{
Actividade Antioxidante en Carne de Tenreiros Alimentados con Bagazo de Uva
}

Recibido: 12 decembro 2017 / Aceptado: 31 outubro 2018

(C) Universidade de Santiago de Compostela 2018

Resumo Os subprodutos de elaboración do viño conteñen valiosos compostos bioactivos, entre os que destacan os polifenois. Os efectos beneficiosos destes compostos atribúense, entre outras, á súa actividade antioxidante. Por esta razón, estúdase o efecto potencial dunha dieta enriquecida en polifenois nas características da carne de tenreiros alimentados con bagazo de uva como complemento ou suplemento na súa alimentación. Proponse unha extracción rápida de seis polifenois individuais de carne crúa de tenreiro, previamente identificados e seleccionados como marcadores no bagazo. O procedemento baséase na extracción simultánea automatizada mediante líquidos presurizados (PLE) seguida de cromatografía líquida de alta eficacia con detección UV-Vis cun sistema de díodos (HPLC-DAD) para a identificación dos polifenois e a análise cuantitativo. Nos extractos obtidos determínanse o índice de polifenois totais (IPT) e a actividade antioxidante (AA). Debido á complexidade da mostra, os resultados máis sobresaíntes deste traballo son os relacionados coa avaliación da actividade antioxidante dos extractos. Tras a

Laura Rubio · Juan Pablo Lamas · Carmen García-Jares · Marta Lores

Laboratorio de Investigación e Desenvolvemento de Solucións Analíticas. Departamento de Química Analítica, Nutrición e Bromatoloxía. Universidade de Santiago de Compostela, Campus VIDA, E-15782, Santiago de Compostela.

Email: laura.rubio@rai.usc.es

Telefono: 881814379; Fax: 881814468

Teresa Moreno

Fundación Centro Tecnolóxico da Carne. Parque Tecnolóxico de Galicia. San Cibrao das Viñas E-32900, Ourense. correspondente análise estatística do conxunto de datos obtidos, pode observarse que resulta mellor incluír unha combinación de bagazo na dieta dos animais dada a mellora observada nos índices antioxidantes medidos, e en consecuencia, tamén nas propiedades da carne e na súa conservación.

Palabras clave Residuos vitivinícolas - Compostos polifenólicos · Extracción con Líquidos Presurizados (PLE) · Índices espectrofotométricos · Matriz complexa.

\section{Antioxidant Activity in Grape-marc-fed Calf Meat}

Abstract Winemaking byproducts contain polyphenols among other valuable bioactive compounds. The beneficial effects of these composts are attributed, among other things, to their antioxidant activity. For this reason, this work studies the potential effect of a diet enriched in polyphenols in the characteristics of calf meat fed with grape bagasse as a supplement to its diet. We propose a rapid extraction of six individual polyphenols of raw calf meat, previously identified and selected as markers in the bagasse. The procedure is based on automated simultaneous extraction using pressurized liquids (PLE) followed by high efficiency liquid chromatography with UV-Vis detection with a diode system (HPLC-DAD) for the identification of polyphenols and quantitative analysis. In the obtained extracts, the total polyphenol index (TP) and the antioxidant activity (AA) are determined. Due to the complexity of the sample, the most outstanding results of this work are those related to the evaluation of the antioxidant activity of the extracts. After the corresponding statistical analysis of the data obtained, it can be observed that it is better to include a combination of grape bagasse in the diet of the animals given the improvement observed in the measured antioxidant indexes, and consequently, also in the properties of the meat and in its conservatione.

Key words Winery byproducts · Polyphenolic compounds · Pressurized Liquid Extraction (PLE) - Spectrophotometric indexes · Complex matrix. 


\section{Introdución}

A carne e os produtos cárnicos son consumidos habitualmente por unha grande parte da poboación. Estase dirixindo a atención á alimentación do animal, dado que dela dependen o seu benestar e a súa saúde, así como a composición e calidade nutritiva dos produtos derivados deles e, en consecuencia, tamén a saúde da poboación. A alimentación dos animais require pois un control da súa composición e calidade. Contémplase a opción de agregar antioxidantes na manutención animal, para conseguir así mellorar a calidade nutritiva e minimizar os procesos de oxidación na carne; para que presente, deste xeito, unhas características sensoriais adecuadas, sexa segura, adaptada para o consumidor e teña unha vida útil prolongada. Hai dúas categorías de antioxidantes en función da súa orixe: naturais (como ácido ascórbico, carotenos ou polifenois) e sintéticos, sendo os primeiros moito máis aceptados polo consumidor (Toner, 2004; Pokorny, 2007).

Por outra banda, a sociedade cada vez amosa maior preocupación pola reciclaxe e aproveitamento dos residuos de orixe natural, co obxectivo de maximizar o rendemento económico (a súa eliminación implica un custe adicional, Devesa Rey et al. 2011) e minimizar o impacto medioambiental. Inténtase así recuperar e reciclar estes subprodutos buscándolle novas aplicacións. Os residuos vexetais da industria agroalimentaria conteñen considerables cantidades de sustancias potencialmente interesantes e, ao mesmo tempo, débese compensar o custo da súa recuperación. Con este propósito, estanse desenvolvendo procesos que pretenden converter estes residuos en biocombustibles, ingredientes en alimentos, así como fontes para a extracción de produtos químicos de alto valor engadido (Fontana et al. 2013; Teixeira et al. 2014). No caso concreto do proceso de elaboración de viño, xéranse subprodutos e residuos ricos en polifenois e a súa extracción é un exemplo desta reutilización á que se fai referencia. Así, enténdese por bagazo de uva a materia residual despois de ser extraído o seu xugo, constituído por pel, pebidas e os cabos dos acios. Como a concentración polifenólica da uva se distribúe entre a polpa (10\%), a pel $(28-35 \%)$ e as pebidas $(60-70 \%)$ (Nerantzis \& Tataridis, 2006), é evidente que unha gran parte dos polifenois quedan no bagazo despois da prensada, sendo este unha fonte relevante deste grupo de compostos. Estes polifenois, metabolitos secundarios biosintetizados no reino vexetal, son compostos bioactivos naturais (Gharras, 2009; Quideau et al. 2011) e presentan actividade antioxidante (García Bacallao et al. 2001; González-Centeno et al. 2013; Álvarez-Casas et al. 2014) entre outras características beneficiosas para a saúde, por isto poden ser utilizados para diversos fins na industria farmacéutica, cosmética e alimentaria.

No tocante ao sector alimentario, os polifenois son utilizados como antioxidantes naturais como suplemento para a alimentación animal, tanto para mellorar a saúde dos animais como para protexer os produtos de orixe animal fronte ao deterioro, como axentes antimicrobianos nos alimentos e como ingredientes funcionais en diversos alimentos e suplementos dietéticos para a alimentación humana (Gharras, 2009).

Así pois, este traballo céntrase na avaliación da actividade antioxidante dos extractos obtidos de carne de tenreiro matriz moi complexa-, tras a inclusión de bagazo seco, unha recoñecida fonte de polifenois, na dieta dos animais. Outros autores empregaron técnicas de extracción en carne para esteroides (Seo et al. 2005) ou sulfonamidas (Gentili et al. 2004) pero non hai antecedentes da aplicación de técnicas de extracción de compostos polifenólicos en este tipo de mostras. É por isto que se decide desenvolver unha nova metodoloxía analítica que permita a extracción e posterior caracterización de polifenois en mostras de carne.

Así, a técnica de extracción seleccionada foi a extracción con líquidos presurizados (PLE) introducida la primeira vez por Richter et al. (1996). O seu fundamento é o uso de disolventes a temperaturas elevadas $\left(50-200^{\circ} \mathrm{C}\right)$ e altas presións (1500-2000 psi), para extraer rápida e eficazmente analitos de matrices sólidas ou semisólidas. Na PLE, o efecto principal da aplicación de elevadas presións é precisamente poder traballar con disolventes en fase líquida a temperaturas por enriba do seu punto de ebulición (Ramos, 2012), que xogan un papel fundamental na mellora da extracción: incrementando a capacidade de solubilizar, aumentando as velocidades de difusión... A natureza do disolvente tamén inflúe na eficacia xa que debe favorecer a solubilidade dos analitos. Entre as vantaxes desta técnica, atópanse a redución do tempo de extracción e o baixo consumo de disolventes orgánicos (Shi et al. 2005; Mendiola et al. 2007) polo que é recoñecida coma unha técnica de extracción verde. Presenta boas condicións de recuperación e precisión, ademais da posibilidade de automatización. A pesar destas vantaxes, unha das limitacións é o elevado custo da instrumentación (Giergielewicz-Możajska et al. 2001). As súas principais áreas de aplicación céntranse no análise medioambiental e na extracción de compoñentes nutricionais e bioactivos en alimentos (Sun et al. 2012).

Este traballo propón, en definitiva, a obtención de extractos rápidos mediante $\mathrm{PLE}$ de carne crúa de tenreiros alimentados con bagazo, para avaliar en termos comparativos o índice de polifenois totais (IPT), a actividade antioxidante (AA) e os seis polifenolis individuais seleccionados como marcadores fronte á carne dun grupo de tenreiros control sen aporte de bagazo na súa alimentación.

\section{Materiais e métodos}

\section{Reactivos}

O material empregado como fase dispersante na preparación de mostra para PLE foi area (200-300 $\mu \mathrm{m}$, Scharlau). Os disolventes de extracción utilizados foron metanol grado HPLC (Panreac) e auga ultra pura producida no laboratorio cun sistema Milli-Q; o eluínte e a fase móbil acidificáronse con ácido acético e fórmico (98\%), 
respectivamente, subministrados por Merck. O reactivo Folin \& Ciocalteau (FC) obtívose de Sigma. Outros produtos químicos usados na determinación dos índices espectrofotométricos foron DPPH (2,2-difenil-1picrilhidrazilo, Sigma) e carbonato sódico $\left(\mathrm{Na}_{2} \mathrm{CO}_{3}\right.$, Panreac). Empregáronse estándares polifenólicos puros para construír as curvas de calibrado: Ácido Gálico monohidratado 99\% (CAS 5895-86-8), Ácido Protocatéquico $>97 \%$ (CAS 99-50-3), Catequina $>98 \%$ (CAS 225937-10-0), Epicatequina $>90 \%$ (CAS 490-46-0), Kaempferol $>90 \%$ (CAS 520-18-3) e Quercetina > 95\% (CAS 117-39-5), todos foron adquiridos en Sigma-Aldrich.

\section{Toma de mostras}

As mostras de carne proveñen do Centro Tecnolóxico da Carne (CTC), fundación galega que pretende dinamizar e mellorar a capacidade competitiva do sector agroalimentario de Galicia, coa que se colabora neste proxecto e onde se leva a cabo a cría e alimentación dos tenreiros segundo un deseño experimental adecuado, así como a recolección das mostras pertinentes. A dieta dos tenreiros inclúe unha base de penso bovino á que se lle engaden herba seca e/ou bagazo como suplementos. Na Táboa 1 amósase a caracterización da alimentación segundo a súa composición analítica, sendo os datos achegados polo CTC, posto que a determinación se realizou nun laboratorio externo, a excepción dos dous derradeiros índices. As mostras recíbense envasadas ao baleiro de maneira individualizada. Analizáronse 20 mostras de carne diferentes, pertencendo a catro grupos claramente diferenciados segundo os complementos subministrados á dieta dos animais: 1. Todo bagazo (codificada como $100 \%$ ); 2 . Todo herba seca (0\%); 3. $1 / 3$ bagazo e $2 / 3$ herba seca (33 \%); e 4 . $2 / 3$ bagazo e $1 / 3$ herba seca $(66 \%)$. Así, analízanse 5 mostras de cada un dos tratamentos, sendo as do grupo 2 as mostras de control, dado que eses tenreiros non consumiron bagazo. Gárdanse a $-20^{\circ} \mathrm{C}$ ata o momento no que van ser empregadas para o seu análise, minimizando así posibles degradacións tanto da carne como dos compostos que poidan conter.

Determinouse tamén a humidade das mostras de carne baseándose na perda de auga polo efecto do quecemento nunha estufa durante $24 \mathrm{~h}$ a unha temperatura entre $100 \mathrm{e}$ $105^{\circ} \mathrm{C}$ ata alcanzar peso constante.

\section{Procedementos analíticos}

Extracción de polifenois: A extracción realizouse mediante a técnica de extracción con líquidos presurizados (PLE) cun equipo da marca BUCHI Speed-Extractor E-916 que permite a extracción simultánea e automatizada de grupos de seis mostras. A mostra de carne e o dispersante (area) nunha relación 1:3, móense nun morteiro de vidro para favorecer a rotura dos tecidos e a posterior extracción dos compostos fenólicos. Esta mestura deposítase na celda de extracción sobre $1 \mathrm{~g}$ de area e cóbrese tamén con area para completar o volume morto da celda. Introdúcense as celdas $(10 \mathrm{~mL})$ no sistema de PLE e extráense cun determinado porcentaxe de disolvente orgánico (metanol) en auga, concretamente, metanol:auga (80:20 v/v) acidificado ao $1 \%$ con ácido acético. As condicións da extracción foron $105^{\circ} \mathrm{C}$, 100 bar de presión e un total de dous ciclos. Os eluatos obtidos enrasáronse a $50 \mathrm{~mL}$ cunha mestura dos disolventes empregados na mesma proporción, fíltranse con filtros de $0,22 \mu \mathrm{m}$ e almacénanse a $-20^{\circ} \mathrm{C}$ ata a realización das determinación analíticas. Realízase a extracción por duplicado de cada unha das mostras.

Análise cromatográfica: Os extractos analizáronse por HPLC nun sistema Jasco equipado cunha columna Phenomenex Kinetex C18 (4,6 x 150 mm; 5 m; $100 \AA)$ e detector de rede de díodos (DAD) con inxector automático. O volume de inxección foi de $5 \mu \mathrm{L}$ sendo constituíntes da fase móbil (A) 0,1\% ácido fórmico en auga e (B) 0,1\% ácido fórmico en metanol. O gradiente programado comezou en $5 \%$ B, cambiou a $20 \%$ B aos $10 \mathrm{~min}$, a $50 \%$ B aos $20 \mathrm{~min}$, a $100 \% \mathrm{~B}$ aos $25 \mathrm{~min}$ e finalmente regresando a $5 \%$ B aos $35 \mathrm{~min}$, onde se mantén 5 min máis. O tempo de análise foi de $40 \mathrm{~min}$ a un fluxo de $1 \mathrm{~mL} \cdot \mathrm{min}^{-1}$ e a unha temperatura constante de $50^{\circ} \mathrm{C}$. A identificación dos diferentes polifenois levouse a cabo a diferentes lonxitudes de onda no rango comprendido entre 200 e 600 nm, ás que absorben eses compostos en función da súa estrutura. A partir da biblioteca de espectros UV e cunha selección de patróns comerciais, comparáranse os espectros e os tempos de retención de ditos patróns cos picos cromatográficos obtidos na inxección dos extractos.

\begin{tabular}{lccc}
\hline \multirow{2}{*}{ DETERMINACIÓNS } & \multicolumn{2}{c}{ ALIMENTOS incluídos na dieta dos tenreiros } \\
\cline { 2 - 4 } & PENSO & Herba seca & BUPLEMENTOS \\
\cline { 2 - 4 } & & - & - \\
\hline Amidón (\%) & 37,90 & 6,50 & 7,89 \\
\hline Cinzas brutas (\%) & 5,51 & 33,40 & 4,45 \\
\hline Fibra bruta (\%) & 4,70 & - & 9,89 \\
\hline Graxa bruta (\%) & 4,73 & 3,64 & 82,45 \\
\hline Humidade (\%) & 12,40 & 93,7 & - \\
\hline Materia orgánica (\%) & - & 89,05 & 12,86 \\
\hline Materia seca (\%) & - & 6,20 & 17,07 \\
\hline Proteína bruta (\%) & 13,08 & - & 3,26 \\
\hline IPT (mg gálico/g extracto seco) & - & - & \\
\hline AA (mM Trolox/g extracto seco) & - & & \\
\hline
\end{tabular}

Táboa 1.- Caracterización dos alimentos incluídos na dieta dos tenreiros segundo a composición analítica dos mesmos 


\begin{tabular}{|c|c|c|c|c|c|c|}
\hline \multirow[t]{2}{*}{ COMPOSTO } & \multirow[t]{2}{*}{ Recta de calibrado } & \multirow[t]{2}{*}{$\mathrm{R}^{2}$} & \multicolumn{2}{|c|}{ Mostra 1} & \multicolumn{2}{|c|}{ Mostra 2} \\
\hline & & & $\mathrm{ppm}$ & $\%$ & $\mathrm{ppm}$ & $\%$ \\
\hline Ácido Gálico & $y=12677 x-36877$ & 0,9993 & 20,91 & 73,44 & 20,57 & 72,23 \\
\hline Ácido Protocatéquico & $y=9490,2 x-26067$ & 0,9993 & 21,29 & 74,75 & 21,29 & 74,75 \\
\hline Catequina & $y=3153 x-11184$ & 0,9992 & 22,48 & 72,06 & 21,88 & 70,14 \\
\hline Epicatequina & $y=3392,9 x-12054$ & 0,9992 & 22,46 & 72,00 & 21,95 & 70,36 \\
\hline Quercetina & $y=6726,2 x-34082$ & 0,9991 & 20,10 & 74,10 & 19,94 & 73,53 \\
\hline Kaempferol & $y=9698,2 x-39547$ & 0,9992 & 21,31 & 74,83 & 21,46 & 75,35 \\
\hline
\end{tabular}

Táboa 2.- Ecuacións das rectas de calibrado e recuperacións para os seis compostos estudados

Cuantificación: As curvas de calibrado dos polifenois seleccionados realízanse nun rango de concentración de 10-100 mg.L-1 (ppm), obténdose as ecuacións reflexadas na Táboa 2. A linearidade instrumental resultou satisfactoria no rango de concentración estudado, con coeficientes de correlación que van dende 0,9991 ata 0,9993.

\section{Medidas espectrofotométricas}

Índice de Polifenois Totais (IPT): A determinación de polifenois totais levouse a cabo por triplicado a cada unha das mostras, mediante o procedemento descrito anteriormente por Singleton \& Rossi (1965). Mesturáronse 5 $\mathrm{mL}$ de disolución acuosa de extracto (dilución 1:10), $100 \mu \mathrm{L}$ do reactivo $\mathrm{FC}$ e $1 \mathrm{~mL}$ dunha solución acuosa de carbonato de sodio $\left(20 \% \mathrm{Na}_{2} \mathrm{CO}_{3}\right)$. Despois de axitar en vórtex, a mestura de reacción mantívose $30 \mathrm{~min}$ na escuridade a temperatura ambiente, tempo suficiente para a redución do reactivo FC polos compostos polifenólicos en condicións alcalinas, o que resulta no desenrolo dunha cor azul. A medida realízase a $760 \mathrm{~nm}$ (Espectrofotómetro Shimazdu UVmini-1240) contra un branco preparado con auga Milli-Q e cuantifícase a partir dunha curva de calibrado preparada con disolucións estándar de ácido gálico en concentracións que varían de 3 a $20 \mathrm{mg} \cdot \mathrm{L}^{-1}\left(\mathrm{R}^{2}=0.9947\right)$. Os resultados son expresados como $\mathrm{mg}$ de ácido gálico equivalentes no extracto líquido (mg.L-1 GAE) así como mg de ácido gálico por $\mathrm{g}$ de mostra seca. A medida espectrofotométrica de cada mostra realízase por triplicado.

Actividade Antioxidante (AA): A actividade antioxidante dos extractos determinouse co DPPH fronte a Trolox $®$ mediante o método de Brand-Williams et al. (1995). Mestúranse $0,1 \mathrm{~mL}$ do extracto e engádenselle $3,9 \mathrm{~mL}$ da disolución metanólica de DPPH (0,1 mM). Despois de axitar en vórtex vigorosamente, a mestura de reacción mantense na escuridade durante $30 \mathrm{~min}$ a temperatura ambiente, tempo durante o cal mingua a intensidade da cor da disolución. A medida realízase a $515 \mathrm{~nm}$ contra un branco de $\mathrm{MeOH} 100 \%$ (medida de absorbancia máxima) e cuantifícase mediante unha regresión lineal $\left(R^{2}=0,9985\right)$ despois de representar a absorbancia a $515 \mathrm{~nm}$ de nove disolucións coñecidas de Trolox (0,1-1 mM). Por diferenza da absorbancia máxima (en ausencia de compostos antioxidantes) e a absorbancia en presenza destes, obtense o valor que nos relaciona a perda de cor da disolución debido á presenza de compostos antioxidantes, isto é, unha medida da capacidade antioxidante da disolución. Exprésase a $\mathrm{AA}$ dos extractos en $\mathrm{mM}$ Trolox/g de carne (peso seco). Realízase a medida espectrofotométrica de cada mostra por triplicado.

Análise estatística: A análise dos 120 datos obtidos efectúase co software Statgraphics. Realízase unha análise da varianza (ANOVA) para determinar se existen diferenzas significativas entre os grupos de datos en función dos catro diferentes réximes dietéticos dos animais. Trátase dun ANOVA simple dun factor sendo a variable dependente a medida espectrofotométrica (AA ou IPT) e o factor o \% de bagazo incluído na dieta. Polo tanto, lévanse a cabo dous ANOVA simples, un para AA e outro para IPT, sendo o número de observacións 120 para cada un deles e 4 o número de niveis (os diferentes tratamentos mencionados con anterioridade).

\section{Resultados e discusión}

\section{Experimentos preliminares}

Inicialmente, aplícanse distintas técnicas de extracción (MSPD e PLE) ás mostras do bagazo tinto seco que se inclúe na alimentación dos tenreiros, co fin de caracterizar os polifenois presentes, para posteriormente centrar os estudos en carne nestes compostos identificados e seleccionados. Próbanse diferentes ratio mostra /dispersante, natureza e porcentaxe de disolvente orgánico e acidificación ou non do eluínte. Tamén se hidrolizan os extractos anteriores, para conseguir así a liberación de certos compostos, coma antocianinas, que poidan estar presentes conxugados con glícidos.

A figura 1 mostra o cromatograma dun extracto de bagazo obtido nas condicións experimentais finalmente seleccionadas (ver sección de Material e Métodos). Dos compostos identificados (e confirmados adicionalmente por LC-MS), proponse a extracción de seis polifenois representativos de distintas familias: ácido gálico e ácido protocatéquico (ácidos fenólicos, máis concretamente, benzoicos), catequina e epicatequina (flavanois), kaempferol e quercetina (flavonois); as correspondentes estruturas químicas amósanse tamén na figura 1. 


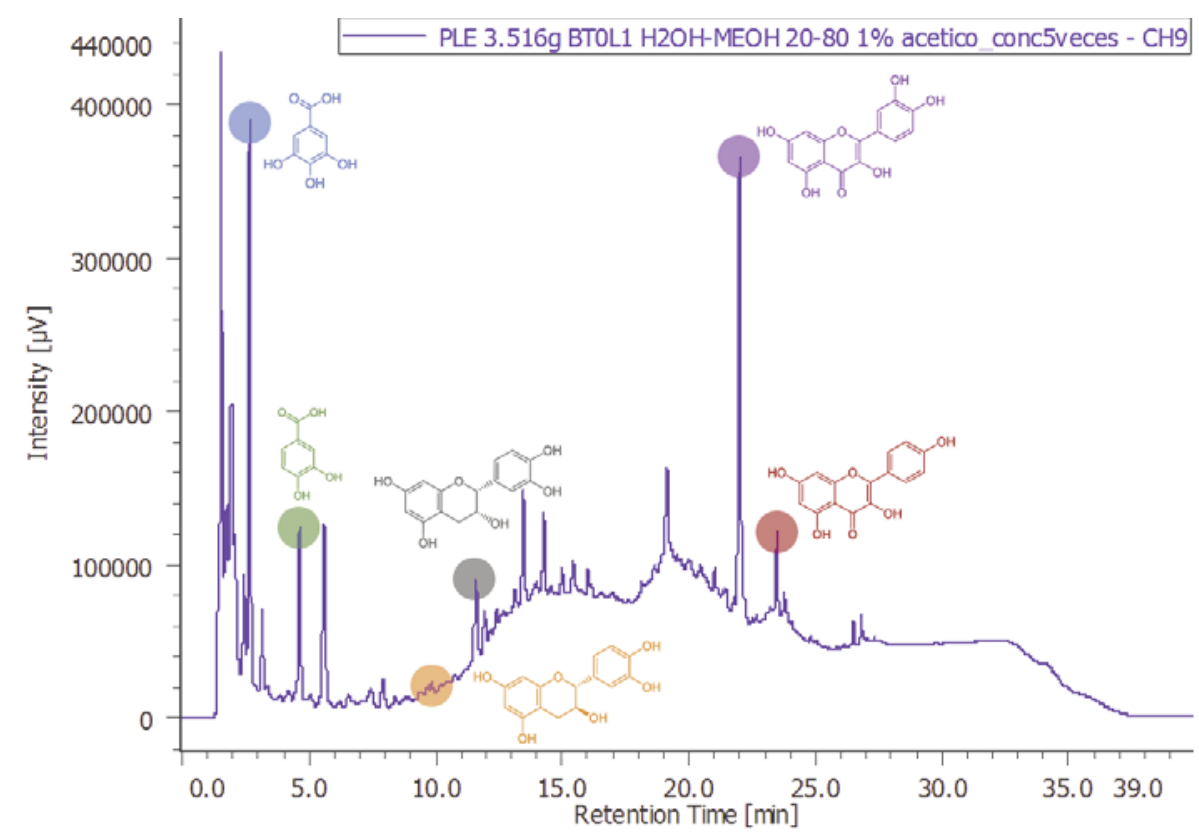

Figura 1.- Cromatograma $(\lambda=250 \mathrm{~nm})$ dun extracto de bagazo (concentrado $5 \mathrm{X}$ ) e estruturas químicas dos seis polifenois seleccionados
Validación do procedemento de extracción en mostras de carne

O CTC proporcionou unha mostra real de carne dun animal non alimentado con bagazo, que se utilizou como matriz para os estudos de recuperación dos polifenois, unha vez confirmada a ausencia dos mesmos mediante a aplicación da metodoloxía proposta PLE-HPLC (Figura 2). De seguido, aplicouse o procedemento a mostras con adición así coma a un branco para realizar un estudo de recuperación. Así, 10 gramos de mostra de carne fortifícanse con $10 \mathrm{~mL}$ dunha disolución que contén entre 200 e $300 \mu \mathrm{g} \cdot \mathrm{mL}^{-1}$ dos 6 polifenois seleccionados (o que equivale a uns $20 \mu \mathrm{g} \cdot \mathrm{mL}^{-1}$ en extracto); tras $24 \mathrm{~h}$ a temperatura ambiente, para que os compostos fenólicos se combinen adecuadamente coa matriz, a mostra almacénanse a $-20^{\circ} \mathrm{C}$. Na análise mediante HPLC identifícanse perfectamente os seis compostos de estudo (Figura 2). E pola súa parte, obtéñense porcentaxes de recuperacións que se atopan nun rango de 71 a $75,50 \%$ (Táboa 2).

\section{Aplicación a mostras reais}

Tras aplicar a metodoloxía analítica PLE-HPLC aos 40 extractos, compróbase a ausencia dos polifenois marcadores en todos eles; tanto na carne de tenreiros control (onde non se esperan) como en todas as mostras de carne de tenreiro alimentados con bagazo en calquera proporción. A hipótese que se contempla é que os polifenois engadidos na dieta se metabolizan na súa totalidade, incrementando o potencial antoxidante da carne (como se demostrará máis adiante) mediante a súa conversión en compostos derivados. O efecto antioxidante directo dun composto diétetico depende da súa absorción no tracto gastrointestinal e deposición nos tecidos (Vasta \& Luciano, 2011). Moitos datos sobre os mecanismos intestinais relacionados coa absorción de polifenois nos ruminantes permanecen descoñecidos.

Se ben é certo que as análises mostran que os polifenois non se detectan como tales na carne, os estudos de contido de polifenois totais ou da actividade antioxidante denontan a presencia de derivados de polifenois (máis concretamente, metabolitos de polifenois) en carne. Descricións similares danse noutros traballos (Jordan et al. 2014; Ortuño et al. 2016), nos cales se atoparon metabolitos de diversos antioxidantes en mostras de carne, mellorando a estabilidade oxidativa desta. Cabe destacar que estes metabolitos conservan os grupos hidroxilo necesarios para interaccionar cos reactivos empregados nas medidas espectrofotométricas, e que así explican os resultados acadados.

Así, tras a análise estatística dos datos obtidos nestas medidas, pode dicirse que existe unha diferenza estatisticamente significativa entre os valores de polifenois totais e actividade antioxidante correspondentes aos extractos de carne dos distintos tratamentos; cun nivel de confianza do $95,0 \%$. A figura 3 amosa as táboas de medias para cada unha das análises, representando as medidas de IPT e AA fronte a \% de bagazo na dieta dos tenreiros. Nos resultados pode observarse que a AA está máis favorecida polo consumo de $2 / 3$ de bagazo ( $1 / 3$ de herba seca); mentres que o IPT está mellorado pola inxesta de 1/3 de bagazo (2/3 de herba seca). En calquera dos casos, resulta mellor unha das combinacións bagazo-herba seca que calquera dos compoñentes por separado. Tamén se podería pensar na hipótese de que ao engadir un exceso destes compostos (e polo tanto, un exceso da cantidade de bagazo na dieta), os animais non consigan asimilalos no seu metabolismo, polo que resultaría mellor unha destas combinacións.

Aquí tamén se pode contemplar a opción de analizar unha mostra da herba seca coa que se alimentan os tenreiros, 
para examinar así se esta contén ou non algún composto polifenólico que poida contribuír a algún destes índices espectrofotométricos. Debido a presenza de polifenois e outros compostos nos vexetais, resulta lóxico que certas herbas teñan un pequeno contido nestes compostos de estudo e se lle atribúan propiedades antioxidantes. A herba procede de praderías polifitas formadas por unha mestura de raigrás inglés, dactilo, Agrostis spp e trevo branco fundamentalmente, aínda que tamén hai outras especies de menor arraigo inicial que van invadindo as praderías conforme teñen xa uns anos. No caso do raigrás inglés
(Lolium perenne) atopáronse nos seus extractos flavonoides glicósidos e ácidos hidroxicinámicos como os polifenois máis abundantes neles (Qawasmeh et al. 2012) e intensidade baixas de glicósidos de Kaempferol (LópezAndrés et al. 2014). Aparecen compostos fenólicos no caso do Dactilo (Dactylis glomerata) (Hauck et al. 2014). Non se atopa nada relevante no caso do Bentgrass (Agrostis spp.), pero si isoflavonas, ácidos fenólicos, flavonoides e clovamidas, entre outros, no Trevo branco (Trifolium repens) (Oleszek et al. 2007; Fooa et al. 2000).

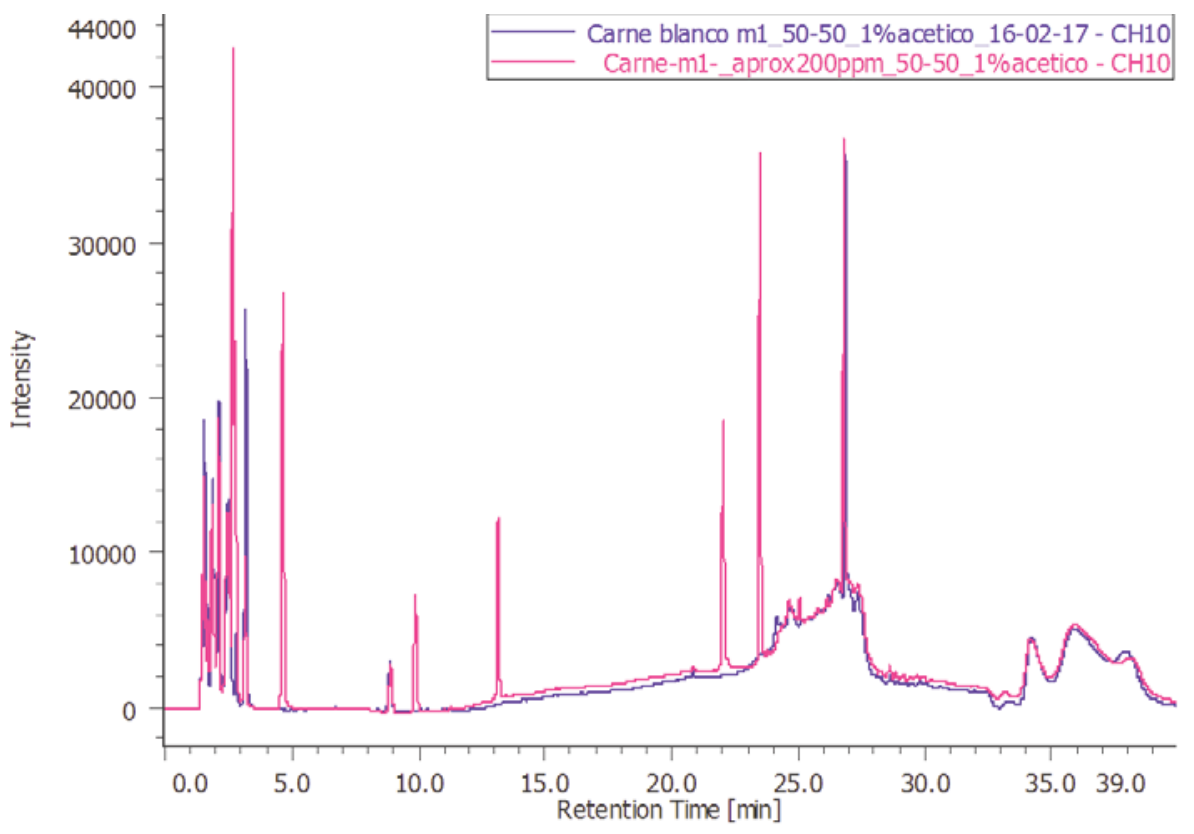

Figura 2.- Cromatogramas $(\lambda=280 \mathrm{~nm})$ correspondentes ao branco da carne (liña azul) e á mostra de carne fortificada cos seis compostos obxectivo (liña rosa)
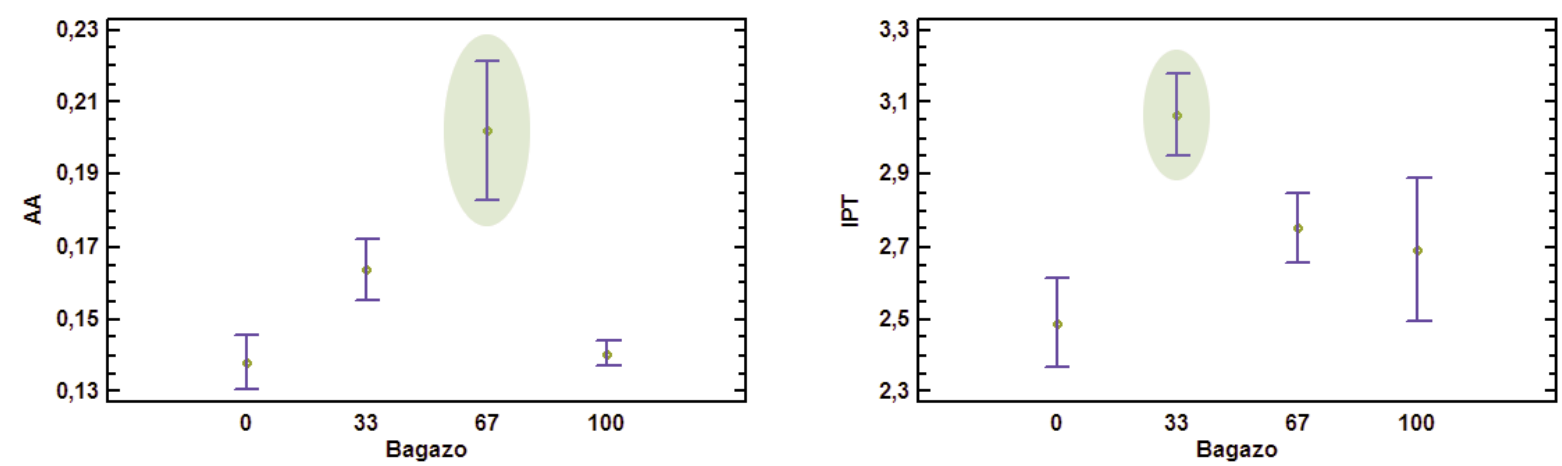

Figura 3.- Valores medios dos dous índices: Actividade antioxidante (AA,esquerda) e polifenois totais (IPT, dereita) das distintas mostras segundo as porcentaxes de bagazo incluídas na dieta. Represéntanse mM Trolox/g extracto seco (AA) e mg gálico/g extracto seco (IPT) fronte a \% de bagazo en ambos casos

Pola súa banda, estas determinacións espectrofotométricas levadas a cabo para a mostra de bagazo incluída na dieta (os resultados amósanse na Táboa 1), demostran o seu contido en polifenois. Pódese explicar así a persistencia dos derivados destes compostos nas mostras de carne e que contribúen aos valores achados. É importante destacar que a incorporación de bagazo das dietas dos animais mellora ambos índices espectrofotométricos. 
Proponse a continuidade desta investigación, con dous obxectivos claros: por unha banda caracterizar o perfil de polifenois dos compoñentes herbáceos da dieta dos tenreiros nas mesmas condicións experimentais que as utilizadas para o bagazo; e, por outra banda, determinar a presenza potencial de metabolitos en fluídos biolóxicos dos tenreiros obxecto de estudo.

\section{Conclusións}

Mediante os estudos aquí realizados non se logrou a detección dos seis polifenois marcadores mediante técnicas cromatográficas, o que se atribúe á metabolización destas sustancias tras o proceso de dixestión. Deste xeito, a estrutura inicial das moléculas vese afectada e non é posible a súa detección como tales. Sen embargo, a metodoloxía analítica desenvolvida resultou satisfactoria para a extracción de polifenois en matrices naturais complexas, tales como a carne; o que se demostra pola obtención de porcentaxes de recuperación axeitadas na validación do procedemento proposto. Por outra banda, o método exposto permite tamén a extracción doutros compostos bioactivos derivados dos polifenois, o que se reflicte no aumento dos valores de IPT dos extractos de carne de tenreiros alimentados con bagazo en calquera proporción fronte ás mostras control. A inclusión de bagazo na dieta dos animais (en combinación coa herba seca) provoca, en consecuencia, unha mellora nos índices antioxidantes, que indica unha melloría nas propiedades antioxidantes da carne que influirá tamén na súa conservación.

Agradecementos Esta investigación foi financiada polo Fondo Europeo de Desenrolo Rexional (FEDER) (2007-2013) e os proxectos RTA2014-0082-C03-01 (INIA_MINECO) UNST13-1E2152, (Programa de Infraestructura, Ministerio de Ciencia e Innovación, España) e GPC2017/04 (Programa de Grupos de Investigación Consolidados, Xunta de Galicia).

\section{Bibliografía}

Álvarez-Casas, M., García-Jares, C., Llompart, M. \& Lores, M. (2014). Effect of experimental parameters in the pressurized solvent extraction of polyphenolic compounds from white grape marc. Food Chemistry. 157, 524-532.

Brand-Williams, W., Cuvelier, M. \& Berset, C. (1995). Use of a free radical method to evaluate antioxidant activity. Food Science and Technology. 28, 25-30.

Devesa Rey, R., Vecino, X., Varela Alende, J. L., Barral, M.T., Cruz, J. M. \& Moldes, A. B. (2011). Valorization of winery waste vs. the costs of no recycling. Waste Management.31, 11: 2327-2335.

Fontana, A. R., Antoniolli, A. \& Bottini, R. (2013). Grape pomace as a sustainable source of bioactive compounds: extraction, characterization, and biotechnological applications of phenolics. Journal of Agricultural and Food Chemistry. 61, 38: 8987-9003.
Fooa, L.Y., Lua, Y., Molanb, A. L., Woodfield, D.R. \& McNabbb, W.C. (2000). The phenols and prodelphinidins of white clover flowers. Phytochemistry. 54, 5: 539-548.

García Bacallao, L., García Gómez, L. V., Rojo Domínguez, D. M. \& Sánchez García, E. (2001). Plantas con propiedades antioxidantes. Revista Cubana de Investigaciones Biomédicas. 20, 3: 231-235.

Gentili, A., Perret, D., Marchese, S., Sergi, M., Olmi, C. \& Curini, R. (2004). Accelerated solvent extraction and confirmatory analysis of sulfonamide residues in raw meat and infant foods by liquid chromatography electrospray tandem mass spectrometry. Journal of Agricultural and Food Chemistry. 52, 15: 4614-4624.

Gharras, H. (2009). Polyphenols: Food sources, properties and applications- a review. International Journal of Food Science and Technology. 44, 12: 2512-2518.

Giergielewicz-Możajska, H., Dąbrowski, Ł. \& Namieśnik, J. (2001). Accelerated Solvent Extraction (ASE) in the analysis of environmental solid samples. Some aspects of theory and practice. Critical Reviews in Analytical Chemistry. 31, 3: 149-165.

González-Centeno M. R., Jourdes, M., Femenia, A., Simal, S., Rosselló, C. \& Teissedre, P. (2013). Characterization of polyphenols and antioxidant potential of white grape pomace byproducts (Vitis vinifera L.). Journal of Agricultural and Food Chemistry. 61, 47: 11579-11587.

Hauck, B., Gallagher, J.A., Morris, S. M., Leemans, D. \& Winters, A. L. (2014). Soluble phenolic compounds in fresh and ensiled orchard grass (Dactylis glomerata L.), a common species in permanent pastures with potential as a biomass feedstock. Journal of Agricultural and Food Chemistry. 62, 2: 468-475.

Jordán, M.J., Castillo, J., Bañón, S., Martínez-Conesa, C. \& Sotomayor, J. A. (2014). Relevance of the carnosic acid/carnosol ratio for the level of rosemary diterpene transfer and for improving lamb meat antioxidant status. Food Chemistry. 151: 212-218.

López-Andrés, P., Luciano ,G., Vasta, V., Gibson, T. M., Scerra, M., Biondi, L., Priolo, A. \& Mueller-Harvey, I. (2014). Antioxidant effects of ryegrass phenolics in lamb liver and plasma. Animal. 8, 1: 51-57.

Mendiola, J.A., Herrero, M., Cifuentes, A. \& Ibañez, E. (2007). Use of compressed fluids for sample preparation: Food applications. Journal of Chromatography A. 1152, 12: $234-246$.

Merken, H.M. \& Beecher G. R. (2000). Measurement of food flavonoids by high-performance liquid chromatography: A review. Journal of Agricultural and Food Chemistry. 48, 3: 577-599.

Naczk, M. \& F. Shahidi. (2004). Extraction and analysis of phenolics in food. Journal of Chromatography A. 1054, 12: $95-111$. 
Nerantzis, E. \& Tataridis, P. (2006). Integrated enologyutilization of winery wastes for the production of high added value products. Journal of Science \& Technology. 1, 3: 7189.

Oleszek, W., Stochmal, A. \& Janda, B. (2007). Concentration of isoflavones and other phenolics in the aerial parts of trifolium species. Journal of Agricultural and Food Chemistry. 55, 20: 8095-8100.

Ortuño, J., Serrano, R., Jordán, M.J. \& Bañón, S. (2016). Relationship between antioxidant status and oxidative stability in lamb meat reinforced with dietary rosemary diterpenes. Food Chemistry. 190: 1056-1063.

Palma, M., Piñeiro, Z. \% Barroso, C. (2001). Stability of phenolic compounds during extraction with superheated solvents. Journal of Chromatography A. 921, 2: 169-174.

Pokorny, J. (2007). Are natural antioxidants better - and safer -than synthetic antioxidants? European Jounal of Lipid Science and Technology. 109, 6: 629-642.

Qawasmeh, A., Obied, H. K., Raman, A. \& Wheatley, W. (2012). Influence of fungal endophyte infection on phenolic content and antioxidant activity in grasses: interaction between Lolium perenne. Journal of Agricultural and Food Chemistry. 60, 13: 3381-3388.

Quideau, S., Deffieux, D., Douat-Casassus, C. \& Pouysegu, L. (2011). Plant polyphenols: chemical properties, biological activities, and synthesis. Angewandte Chemie International Edition. 50, 3: 586- 621.

Ramos, L. (2012). Critical overview of selected contemporary sample preparation technique. Journal of Chromatography A. 1221, 84-98.

Richter, B.E., Jones, B. A., Ezzell, J. L., Porter, N. L., Avdalovic, N. \& Pohl, C. (1996). Accelerated solvent extraction: a technique for sample preparation. Analytical Chemistry. 68, 6: 1033-1039.

Robbins, R.J. (2003). Phenolic Acids in Foods: An Overview of Analytical Methodology. Journal of Agricultural and Food Chemistry. 51, 10: 2866-2887.

Santangelo, C., Varì, R., Scazzocchio, B., Di Benedetto,R., Filesi, C. \& Masella, R. (2007). Polyphenols, intracellular signalling and inflammation. Ann Ist Super Sanita. 43, 4: 394-405.
Seo, J., Kima, H-Y., Chungb, B. C. \& Honga, J. (2005). Simultaneous determination of anabolic steroids and synthetic hormones in meat by freezing-lipid filtration, solid-phase extraction and gas chromatography-mass spectrometry. Journal of Chromatography A. 1067, 1-2: 303-309.

Shi, J., Nawaz, H., Pohorly, J., Mittal, G., Kakuda, Y. \& Jiang, Y. (2005). Extraction of polyphenolics from plant material for functional foods- Engineering and technology. Food Reviews International. 21, 1: 139-166.

Sies, H. (2010). Polyphenols and health: Update and perspectives. Archives of Biochemistry and Biophysics. 501, 1: 2-5.

Singleton, V. L. \& Rossi, J. A. (1965). Colorimetry of total phenolics with phosphomolybdic-phosphotungstic acid reagents. American Journal of Enology and Viticulture. 16, 3: $144-158$

Sun, H., Ge, X.; Lv, Y. \& Wang, A. (2012). Application of accelerated solvent extraction in the analysis of organic contaminants, bioactive and nutritional compounds in food and feed. Journal of Chromatography A. 1237: 1-23.

Teixeira, A., Baenas, N., Domínguez-Perles, R., Barros, A., Rosa, E., Moreno, D. A. \& García-Viguera, C. (2014). Natural bioactive compounds from winery by-products as health promoters: a review. International Journal of Molecular Sciences. 15, 9: 15638-15678.

Toner, C. (2004). Consumer perspectives about antioxidants. The Journal of Nutrition. 134, 11: 3192S3193S.

Vasta, V. \& Luciano, G. (2011). The effects of dietary consumption of plant secondary compounds on small ruminants' products quality. Small Ruminant Research. 101, 1-3: 150-159.

Waterhouse, A.L. (2003). Determination of total phenolics. Current Protocols. Food Analytical Chemistry. 6, 1: 11.1111.18 . 